\title{
Sensitivity Analysis using Data-Driven Parametric Macromodels
}

\author{
Krishnan Chemmangat, Francesco Ferranti, Luc Knockaert, and Tom Dhaene \\ Department of Information Technology, Ghent University - IBBT, \\ Gaston Crommenlaan 8, Bus 201, B-9050, Gent, Belgium. \\ email: $\{$ krishnan.cmc, francesco.ferranti, luc.knockaert, tom.dhaene $\} @$ intec.ugent.be
}

\begin{abstract}
An accurate parametric macromodeling method which builds the parameterized frequency behavior of systems from frequency data samples is presented. The method aims to calculate parametric sensitivity responses of the model with respect to design parameters over the entire design space. A judiciously chosen interpolation scheme is used to parameterize state-space matrices such that parametric sensitivities can be computed analytically. The modeling capability of the proposed method is validated by a pertinent numerical example.
\end{abstract}

\section{Introduction}

The use of parametric macromodels as an approximation of complex systems for design space exploration, design optimization etc., is becoming increasingly important because of their efficiency in terms of reduction in computation time with respect to the original complex system. It is equally important that these parametric macromodels can accurately calculate the parametric sensitivity responses over the entire design space of interest, implying that this information could be used in different stages of the design process such as sensitivity analysis, gradient-based design optimization etc.

One of the most common approaches in calculating local sensitivities is the adjoint variable method $[1,2]$.The main attractiveness of this approach is that sensitivity information can be obtained from at most two systems analyses regardless of the number of designable parameters. However, these methods involve the calculation of system matrix derivatives, which are most frequently estimated by means of finite difference approximations.

In recent years, there has been ongoing research on parametric macromodeling based on the interpolation of systems [3, 4]. These methods interpolate a set of frequency dependent univariate models, called root macromodels, over the parameter space, yielding a complete parametric macromodel. As an extension of these methods, some techniques have been presented in $[5,6]$, where instead of interpolating the root macromod$e l s$, the corresponding state-space matrices are interpolated to obtain a parametric macromodel. These methods allow a parameterization of both poles and residues, thereby providing an improved modeling capability as compared to [3, 4].

This paper proposes a parametric macromodeling technique, which builds parametric sensitivity responses efficiently and accurately over the entire design space of interest. With a proper choice of the interpolation scheme, i.e. at least continuously differentiable, the state-space matrices are parameterized as in $[5,6]$ to build parametric sensitivity macromodels which are able to describe parametric sensitivities analytically. Pertinent numerical results validate the proposed parametric macromodeling approach.

\section{Parametric Sensitivity Macromodeling}

The macromodeling process starts with a set of data samples $\left\{(s, \vec{g})_{k}, \mathbf{H}(s, \vec{g})_{k}\right\}_{k=1}^{K_{t o t}}$ which depends on frequency and several other design parameters. From these data samples, frequency dependent rational models in pole-residue form are built for all grid points in the design space by means of the Vector Fitting (VF) technique [7], yielding the rational model

$$
\mathbf{R}_{\vec{g}_{k}}(s)=\sum_{n=1}^{N_{P}} \frac{\mathbf{c}_{n}^{\vec{g}_{k}}}{s-a_{n}^{\vec{g}_{k}}}+\mathbf{d}^{\vec{g}_{k}}
$$

The rational model in (1) has $N_{P}$ poles with $a_{n}^{\vec{g}_{k}}, \mathbf{c}_{n}^{\vec{g}_{k}}$ and $\mathbf{d}^{\vec{g}_{k}}$ representing poles, residues and the constant term respectively at the design point $\vec{g}_{k}=\left(g_{k_{1}}^{(1)}, \ldots, g_{k_{N}}^{(N)}\right)$. A pole-flipping scheme is used to enforce strict stability [7] and passivity enforcement can be accomplished using one of the robust standard techniques [8,9] resulting in stable and passive rational univariate macromodels called root macromodels.

Each of these root macromodels $\mathbf{R}_{\vec{g}_{k}}(s)$, corresponding to a specific design space point $\vec{g}_{k}$, is converted from a pole-residue form into a state-space form:

$$
\mathbf{R}_{\vec{g}_{k}}(s)=\mathbf{C}_{\vec{g}_{k}}\left(s \mathbf{I}-\mathbf{A}_{\vec{g}_{k}}\right)^{-1} \mathbf{B}_{\vec{g}_{k}}+\mathbf{D}_{\vec{g}_{k}}
$$

The state-space matrices $\mathbf{A}_{\vec{g} k}, \mathbf{B}_{\vec{g} k}, \mathbf{C}_{\vec{g} k}, \mathbf{D}_{\vec{g} k}$ in (2) are parameterized with the help of different interpolation schemes which are at least continuously differentiable over rectangular grids. The continuous differentiability of the interpolation scheme ensures that the derivatives are smooth and sufficiently accurate. Here we have investigated two different interpolation schemes, namely Cubic Spline (CS) interpolation and Piecewise Cubic Hermite Interpolation (PCHIP) which are briefly described in the sequel.

Cubic Spline (CS) Interpolation In this method a cubic spline polynomial $s^{i}(x)$ is built for each interval $x_{i} \leq x \leq x_{i+1}$, $i=1, \ldots, n$ of the input-output data set $\left(x_{i}, y_{i}\right)_{i=1}^{n}$. The coefficients of each of these polynomials are calculated by imposing the first and second order derivative continuity at the data points along with a not-a-knot end condition [10]. Once these coefficients are computed, the derivatives of the overall spline interpolation function can be calculated analytically in terms of its coefficients. If the data to be interpolated happen to be matrices, each entry of the matrices is independently interpolated.

The univariate CS interpolation can be extended to higher dimensions by means of a tensor product implementation [10].

Piecewise Cubic Hermite Interpolation (PCHIP) The PCHIP method is a monotonic shape preserving interpolation scheme. As in the CS interpolation, each data interval is modeled by a cubic polynomial with additional constraints to preserve the monotonicity locally [11]. An extension to higher 
dimension can be performed by a tensor product implementation [10]. As in the CS interpolation case, the derivatives are calculated analytically. Since PCHIP is a bounded interpolation scheme it works better for non-smooth datasets, whereas CS could result in overshoots or oscillatory behavior. However, PCHIP is only continuous in the first derivatives, unlike $\mathrm{CS}$ which is continuous in the second derivatives, which effects the smoothness of the derivatives obtained from PCHIP [11].

\section{Parametric Sensitivity Macromodels}

The set of root macromodel state-space matrices $\mathbf{A}_{\vec{g} k}, \mathbf{B}_{\vec{g} k}$, $\mathbf{C}_{\vec{g} k}, \mathbf{D}_{\vec{g} k}$ is interpolated entry-wise and the multivariate models $\mathbf{A}(\vec{g}), \mathbf{B}(\vec{g}), \mathbf{C}(\vec{g}), \mathbf{D}(\vec{g})$ are built, yielding a parametric macromodel over the entire design space,

$$
\mathbf{R}(s, \vec{g})=\mathbf{C}(\vec{g})(s \mathbf{I}-\mathbf{A}(\vec{g}))^{-1} \mathbf{B}(\vec{g})+\mathbf{D}(\vec{g}) .
$$

A parametric macromodel of sensitivity responses is obtained by differentiating (3) with respect to the design parameters $\vec{g}$, i.e.:

$$
\begin{array}{r}
\frac{\partial}{\partial \vec{g}} \mathbf{R}(s, \vec{g})=\frac{\partial \mathbf{C}(\vec{g})}{\partial \vec{g}}(s \mathbf{I}-\mathbf{A}(\vec{g}))^{-1} \mathbf{B}(\vec{g})+ \\
\mathbf{C}(\vec{g})(s \mathbf{I}-\mathbf{A}(\vec{g}))^{-1} \frac{\partial \mathbf{A}(\vec{g})}{\partial \vec{g}}(s \mathbf{I}-\mathbf{A}(\vec{g}))^{-1} \mathbf{B}(\vec{g})+ \\
\mathbf{C}(\vec{g})(s \mathbf{I}-\mathbf{A}(\vec{g}))^{-1} \frac{\partial \mathbf{B}(\vec{g})}{\partial \vec{g}}+\frac{\partial \mathbf{D}(\vec{g})}{\partial \vec{g}}
\end{array}
$$

In (4), $\frac{\partial}{\partial \vec{g}} \mathbf{R}(s, \vec{g})$ is a function of the parameterized matrices, $\mathbf{A}(\vec{g}), \mathbf{B}(\vec{g}), \mathbf{C}(\vec{g}), \mathbf{D}(\vec{g})$ and their derivatives, computed efficiently and analytically using the CS and PCHIP interpolation schemes.

A schematic of the proposed method is shown in Fig. 1.

\section{Numerical Example}

A coaxial cable is modeled with cross section shown in Fig. 2. The relative permittivity $\epsilon_{r e l}$ of the dielectric is chosen equal to 2.5. The impedance matrix $Z(s, a, L)$ of the model is calculated analytically [12] as a function of the radius of the inner conductor $a$ and the length $L$, in addition to frequency, on a grid of $150 \times 15 \times 15$ samples (freq, $a, L)$. The corresponding ranges of these parameters are shown in Table 1.

\begin{tabular}{|l|c|c|}
\hline Parameter & Min & Max \\
\hline Frequency $($ freq $)$ & $10 \mathrm{KHz}$ & $2 \mathrm{GHz}$ \\
Inner radius $(a)$ & $2 \mathrm{~mm}$ & $3 \mathrm{~mm}$ \\
Length $(L)$ & $100 \mathrm{~mm}$ & $110 \mathrm{~mm}$ \\
\hline
\end{tabular}

Table 1: Design parameters of the coaxial cable

A set of stable and passive root macromodels has been built for 8 values of $a$ and 8 values of $L$ using VF. The remaining data are used for validation. The number of poles $N_{P}$ for each root macromodel is 18, selected using an error-based bottom up approach. Each root macromodel has been converted to statespace form and the state-space matrices have been interpolated by the CS and PCHIP interpolation methods. Next, the parametric sensitivity of $Z(s, a, L)$ with respect to $a$ and $L$ has

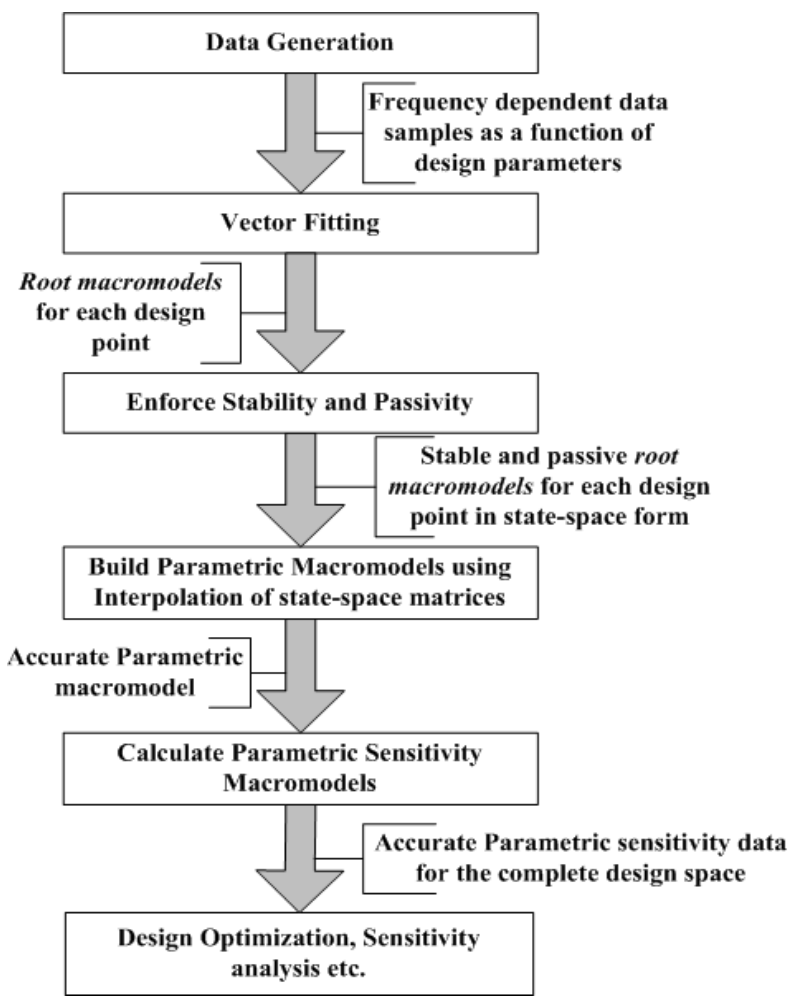

Figure 1: Schematic of parametric sensitivity macromodeling.

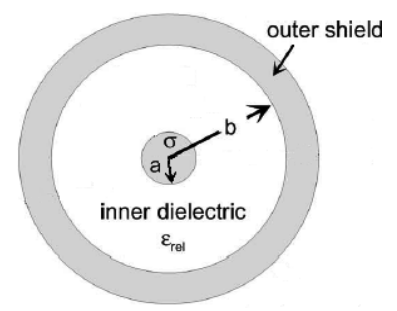

Figure 2: Cross section of the coaxial cable.

been computed by means of the derivatives (4) of the trivariate macromodel and the analytical model computed from [12]. The accuracy of the model and its derivatives for the two interpolation methods are measured in terms of the weighted rms-error defined as:

$\mathrm{E}_{\mathrm{RMS}}(\vec{g})=\sqrt{\frac{\sum_{i=1}^{P^{2}} \sum_{k=1}^{K_{s}}\left|w_{Z_{i}}(s, \vec{g})\left(R_{i}\left(s_{k}, \vec{g}\right)-Z_{i}\left(s_{k}, \vec{g}\right)\right)\right|^{2}}{P^{2} K_{s}}}$.

In (5) $P$ is the number of ports, $K_{s}$ is the number of frequency samples and $w_{Z_{i}}=\left|Z_{i}\left(s_{k}, \vec{g}\right)\right|^{-1}$ is the weighting function for the error. The worst case rms-error over the validation grid is chosen to assess the accuracy and the quality of the parametric sensitivity macromodels

$$
\mathrm{E}_{\mathrm{RMS}}^{\mathrm{Max}}=\max _{\vec{g}} \mathrm{E}_{\mathrm{RMS}}(\vec{g}), \vec{g} \in \text { validation grid }
$$

The maximum weighted rms-error calculated from (6) for the model and its sensitivities is tabulated in Table 2. 


\begin{tabular}{|c|c|c|}
\hline \multirow{2}{*}{ Quantity } & \multicolumn{2}{|c|}{ Method } \\
\cline { 2 - 3 } & CS & PCHIP \\
\hline $\mathbf{Z}(s, a, L)$ & 0.0054 & 0.0054 \\
\hline$\frac{\partial \mathbf{Z}(s, a, L)}{\partial a}$ & 0.0061 & 0.0325 \\
\hline$\frac{\partial \mathbf{Z}(s, a, L)}{\partial L}$ & 0.0119 & 0.0194 \\
\hline
\end{tabular}

Table 2: Modeling accuracy of the proposed method

Fig. 3 shows the magnitude of $Z_{11}$ as a function of frequency and $a$ for $L=105 \mathrm{~mm}$, while Fig. 4 shows the magnitude of the corresponding parametric sensitivity $\frac{\partial \mathbf{Z}_{11}}{\partial a}$ obtained by the CS scheme. Fig. 5 shows the magnitude of $Z_{12}$ as a function of frequency and $L$ for $a=2.5 \mathrm{~mm}$, while Fig. 6 shows the magnitude of the corresponding parametric sensitivity $\frac{\partial \mathbf{Z}_{12}}{\partial L}$ obtained by the CS scheme. Fig. 7 compares the magnitude of $\frac{\partial \mathbf{Z}_{11}}{\partial a}$ obtained by the analytical model, and the CS and PCHIP methods for the values $a=2.5 \mathrm{~mm}$ and $L=105 \mathrm{~mm}$, which have not been used for the generation of the root macromodels. Fig. 8 shows the magnitude of $\frac{\partial \mathbf{Z}_{12}}{\partial L}$ for the same values of $a$ and $L$. A very good agreement between the methods can be observed.

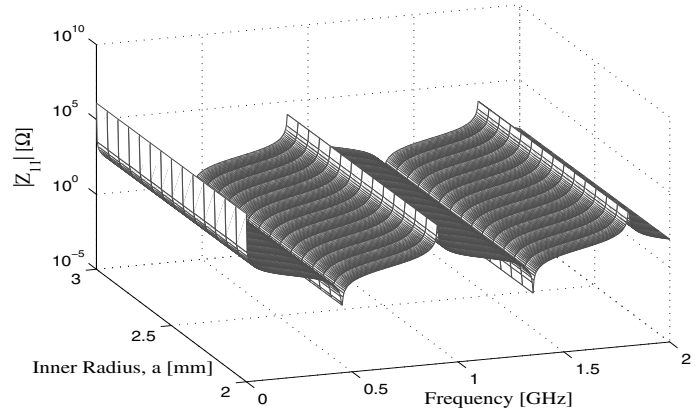

Figure 3: Magnitude of $Z_{11}$ for $L=105 \mathrm{~mm}$.

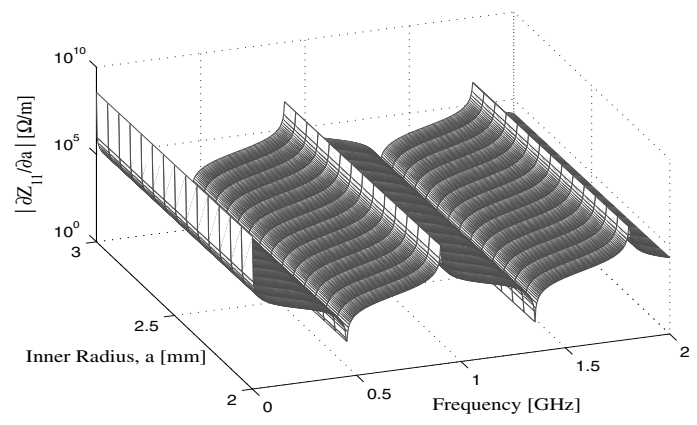

Figure 4: Magnitude of $\frac{\partial Z_{11}}{\partial a}(\mathrm{CS})$ for $L=105 \mathrm{~mm}$.

Fig.9 shows the rms-error distribution of the parametric macromodel using the CS interpolation scheme with respect to the analytical model of [12] over the complete design space.

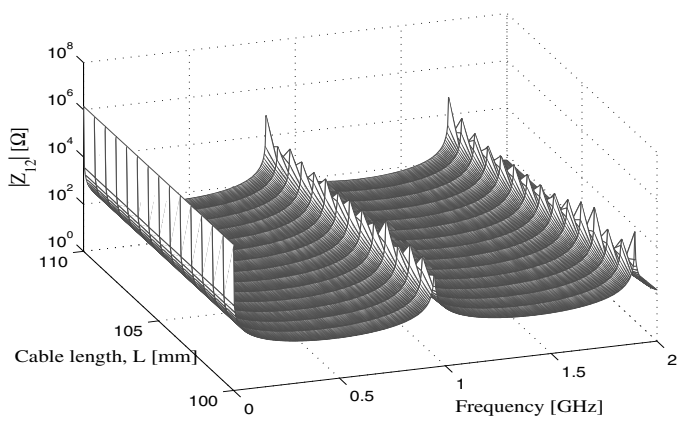

Figure 5: Magnitude of $Z_{12}$ for $a=2.5 \mathrm{~mm}$.

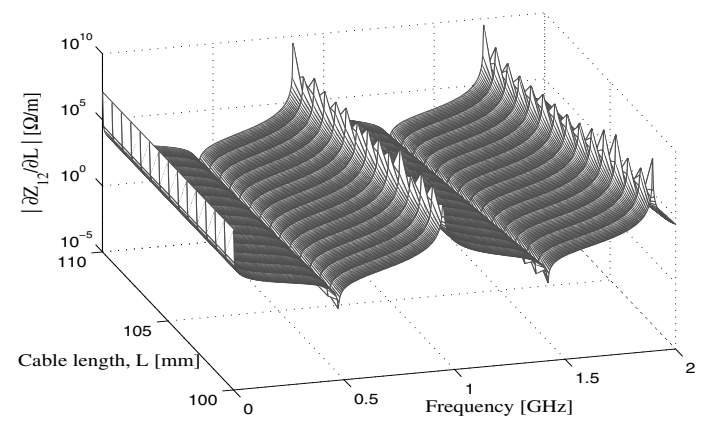

Figure 6: Magnitude of $\frac{\partial Z_{12}}{\partial L}(\mathrm{CS})$ for $a=2.5 \mathrm{~mm}$.

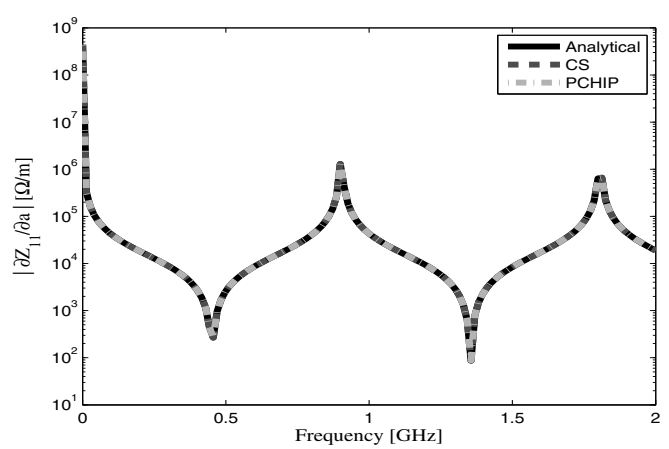

Figure 7: Magnitude of $\frac{\partial Z_{11}}{\partial a}$ for $a=2.5 \mathrm{~mm}$ and $L=105$ $m m$. 


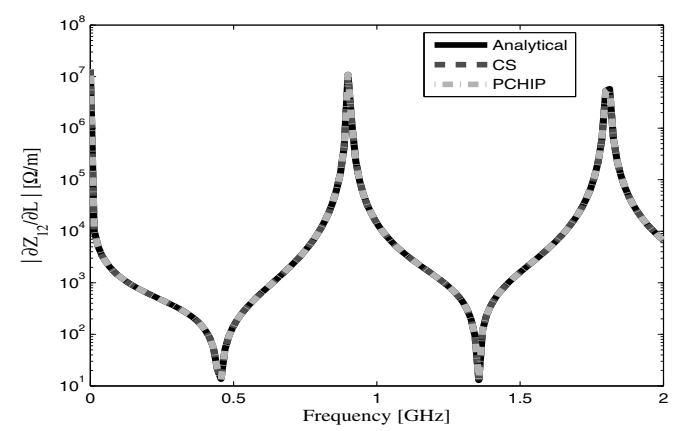

Figure 8: Magnitude of $\frac{\partial Z_{12}}{\partial L}$ for $a=2.5 \mathrm{~mm}$ and $L=105$ $m m$.

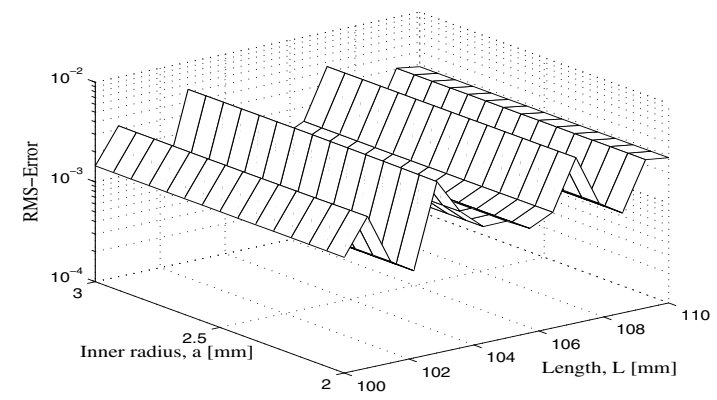

Figure 9: RMS-Error of $Z$ for the entire design space (CS).

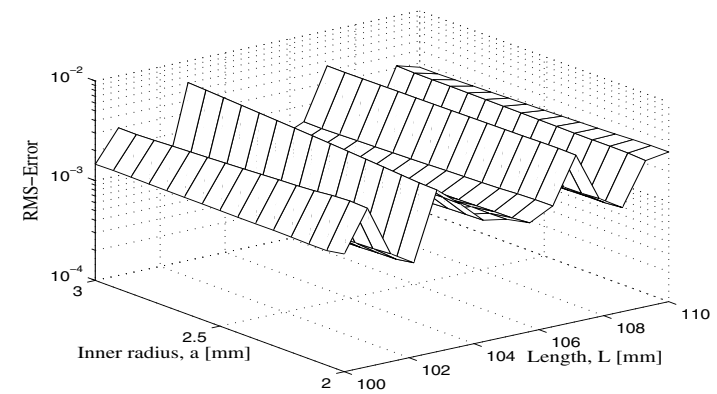

Figure 10: RMS-Error of $\frac{\partial Z}{\partial L}$ for the entire design space (CS).

Fig.10 shows the rms-error distribution of the parametric sensitivity macromodel of $\frac{\partial Z}{\partial L}$ with respect to the analytical model. Similar results were obtained for other cases in Table 2 . We note that a good accuracy is achieved by both interpolation methods, but the CS scheme leads to a lower average error, probably due to the continuity of the second derivatives.

\section{Conclusions}

This paper presents a new macromodeling technique for accurately calculating parametric sensitivity responses from frequency sampled data. The parameterization of the model and calculation of the parametric sensitivities is based on the interpolation of state-space matrices with a judicious choice of the interpolation scheme. The proposed method has been validated using a pertinent numerical example, thereby demonstrating its modeling capability.

\section{Acknowledgments}

This work was supported by the Research Foundation Flanders (FWO).

\section{References}

[1] Li, D. Zhu, J. Nikolova, N.K. Bakr, M.H. and Bandler, J.W., "Electromagnetic optimisation using sensitivity analysis in the frequency domain," Microwaves, Antennas Propagation, IET, Vol. 1, No. 4 (2007), pp. 852 -859.

[2] Dounavis, A. Achar, R. and Nakhla M.S., "Efficient Sensitivity Analysis of Lossy Multiconductor Transmission Lines With Nonlinear Terminations," IEEE Transactions on Microwave Theory and Techniques, Vol. 49, No. 12 (2001), pp. 2292-2299.

[3] Ferranti, F. Knockaert, L. and Dhaene, T., "Parameterized S-Parameter Based Macromodeling With Guaranteed Passivity," IEEE Microwave and Wireless Components Letters, Vol. 19, No. 10 (2009), pp. 608-610.

[4] Ferranti, F. Knockaert, L. and Dhaene, T., "Guaranteed Passive Parameterized Admittance-Based Macromodeling," IEEE Transactions on Advanced Packaging, Vol. 33, No. 3 (2010), pp. 623-629.

[5] Ferranti, F. Knockaert, L. Dhaene, T. and Antonini, G., "Passivity-Preserving Parametric Macromodeling for Highly Dynamic Tabulated Data Based on Lur'e Equations," IEEE Transactions on Microwave Theory and Techniques, Vol. 58, No. 12 (2010), pp. 3688-3696.

[6] Triverio, P. Nakhla, M.S. and Grivet-Talocia, S., "Passive parametric macromodeling from sampled frequency data," Signal Propagation on Interconnects (SPI), 2010 IEEE 14th Workshop on, (2010), pp. 117-120.

[7] Gustavsen, B. and Semlyen, A., "Rational approximation of frequency domain responses by Vector Fitting," IEEE Transactions on Power Delivery, Vol. 14, No. 3 (1999), pp. 1052-1061.

[8] Gustavsen, B. and Semlyen, A., "A Half-Size Singularity Test Matrix for Fast and Reliable Passivity Assessment of Rational Models," IEEE Transactions on Power Delivery, Vol. 24, No. 1 (2009), pp. 345-351.

[9] Gustavsen, B., "Fast passivity enforcement for pole-residue models by perturbation of residue matrix eigenvalues," IEEE Transactions on Power Delivery, Vol. 23, No. 4 (2008), pp. 2278-2285.

[10] de Boor, C. et al., A Practical Guide to Splines, SpringerVerlagl (New York, 2001).

[11] Fritsch, F. N. and Carlson, R. E., "Monotone Piecewise Cubic Interpolation," SIAM Journal on Numerical Analysis, Vol. 17, No. 2 (1980), pp. 238-246.

[12] Tesche, M.F., "A simple model for the line parameters of a lossy coaxial cable filled with a nondispersive dielectric," IEEE Transactions on Electromagnetic Compatibility, Vol. 49, No. 1 (2007), pp. 12-17. 